\title{
Vague nerve stimulation in chronic heart failure: is it worth it?
}

\author{
Mariana Floria ${ }^{*}, 1,2$, Mihaela Grecu ${ }^{3}$, Grigore Tinică ${ }^{2,3}$ \\ ${ }^{1}$ Emergency Hospital lasi, Romania; ${ }^{2}$ Grigore T Popa" University of Medicine and Pharmacy lasi, \\ Romania; ${ }^{3}$ Cardiovascular Disease Institute lasi, Romania
}

\begin{abstract}
In heart failure the major pathophysiology changes are enhanced sympathetic nervous system activity and reduced parasympathetic activation. Despite advances in pharmacologic treatment and interventional therapies such as implantable devices, mortality and morbidity in patients with advanced heart failure is still higher. In the last three decades the novel interventional and device-based therapies aim to restore cardiac autonomic balance by neuromodulation. Chronic vague nerve stimulation (VNS) is a neuromodulator method proposed as a new potential therapy in patients with heart failure. Results of preclinical animal studies and early clinical trials have demonstrated the safety and efficacy of this therapy in heart failure. Although a favorable long-term safety profile was found in human, improvements in the efficacy endpoints in patients with heart failure by VNS are controversially, as demonstrated by one of the largest recently published trial. Despite that VNS does not reduce left ventricular end-systolic volume index, the rate of death or heart failure events in chronic heart failure patients, this new treatment improved quality of life, NYHA functional class, and 6-min walking distance. Large randomized clinical studies are necessary to evaluate the clinical role of this new therapeutic approach in chronic heart failure.
\end{abstract}

Keywords: heart failure, vague nerve stimulation, neuromodulation, minim invasive

\section{Introduction}

In heart failure the major pathophysiology changes are enhanced sympathetic nervous system activity and reduced parasympathetic activation. Despite advances in pharmacologic treatment and non-pharmacological therapies such as cardiac resynchronization therapy, mortality and morbidity in patients with advanced heart failure is still higher, with a rate survival around of $50 \%$ [1].

An autonomic imbalance with decreasing in vagal activity and increasing in sympathetic activity is present in heart failure. Vague nerve

Received: August 2017; Accepted after review: September 2017; Published: September 2017.

*Corresponding author: Mariana Floria, "Grigore T. Popa" University of Medicine and Pharmacy, 16 Universitatii str. 700115, Iasi, Romania.

E-mail: floria mariana@yahoo.com. stimulation (VNS) is a neuromodulation method, aiming to restore the sympatho-vagal balance, without causing permanent damage to the autonomic structures. Chronic VNS was proposed as a new potential therapy in patients with heart failure. Results of preclinical animal studies and early clinical trials have demonstrated the safety and efficacy of these therapies in heart failure [2].

\section{Cardiac autonomic nervous system}

The thoracic vagal nerves have a complex structure. In addition to the parasympathetic structure that sends fibers to various parts of the body, a sympathetic component is known to be present in the vagal nerves based on physiological observations [3]. In experimental studies isolated vagal nerve activation induces tachycardia, due to the activation of the sympathetic component of the vagal nerves [4]. 
The cardiac autonomic nervous system has 2 components (sympathetic and parasympathetic systems) that work in an opposing fashion in the heart [3]. These 2 components exert stimulatory or inhibitory effects on target tissue via adrenergic and muscarinic receptors. Both sympathetic and the parasympathetic branches of the autonomic nervous system are composed of afferent and efferent, as well as interneuronal fibers. Sympathetic innervation originates mainly in the right and left stellate ganglia. These fibers travel along the epicardial vascular structures of the heart into the underlying myocardium and end as sympathetic nerve terminals reaching the endocardium. Parasympathetic effects are carried by the right and left vague nerves, originating in the medulla. The vague nerve further divides into the superior and inferior cardiac nerves, finally merging with the postganglionic sympathetic neurons to form a plexus of nerves at the base of the heart, known as the cardiac plexus [3].

Novel interventional and device-based therapies aim to restore cardiac autonomic balance by neuromodulation. Several devices for selective modulation of sympathetic and vagal activity have recently been developed in an attempt to alter the natural history of heart failure. Therapeutic neuromodulation with device-based therapies, either with vagal stimulation, renal denervation, baroreceptor activation, or left cardiac sympathetic denervation has been used safely in humans since over three decades.

Alterations in cardiac autonomic nervous system function occur in several interrelated cardiac conditions, including hypertension, myocardial ischemia, heart failure, cardiac arrhythmias, and sudden cardiac death.

\section{Vague nerve stimulation in chronic heart failure - experimental data}

Vague nerve stimulation was performed experimentally for the first time more than 150 years ago [5]. Experimental studies have demonstrated that vagal stimulation has an antifibrillatory effect and has proven to be beneficial in animal models of heart failure [ 6 , 7].
Nowadays it is well known the beneficial effect of augmented vagal activity in the setting of chronic heart failure. Stimulating the afferent cervical vagal nerve in experimental models suppresses sympathetic discharges. Because cervical vagal nerves are accessible through surgical approaches, they are the prime target for neuromodulation with the hope that their stimulation will achieve therapeutic effects distant beyond the nerves stimulated.

Studies in experimental models of heart failure showed that chronic VNS exerts beneficial effects on left ventricular systolic function and on survival (by a reduction in mortality rate at 140 days to $14 \%$ with VNS stimulation) [6-8]. Vague nerve stimulation might be also used to attenuate heart failure development in animal models. Mechanisms of action may include the following: heart rate, anti-adrenergic, anti-apoptotic, and antiinflammatory effects as well as an increase in nitric oxide [5].

Although most of experimental studies used stimulus strength sufficient to reduce heart rate, low-level VNS (defined by a stimulus strength $1 \mathrm{~V}$ below the threshold needed to reduce heart rate) is effective in suppressing atrial fibrillation induction in openchest-anesthetized dogs [9]. Vague nerve stimulation may achieve the therapeutic effects by suppressing sympathetic outflow to the heart. However, the effects of VNS don't seem to be permanent [5]. In addition it might induce the remodeling of the stellate ganglion by increasing small-conductance calcium activated $\mathrm{K}$ channel subtype 2 proteins and the reduction of tyrosine hydroxylase-positive ganglion cells [5]. Low-level VNS decreased the density of nerve structures (presumably sympathetic). Although a majority ( $>90 \%)$ of the ganglion cells normally stain positive for tyrosine hydroxylase, a small minority of cells show no tyrosine hydroxylase staining [5]. Vague nerve stimulation might also cause phenotypic switching between adrenergic and cholinergic nerves [5].

\section{Vague nerve stimulation in chronic heart failure - clinical data}

The potential value of chronic VNS was explored in humans with an implantable neurostimulator capable of delivering low current 
pulses with adjustable parameters to stimulate the right (or left cervical) vague. Experimentally stimulation of vagal afferent nerve fibers can have profound effects on the activity of the contralateral vagal efferent (increased activity) and bilaterally of cardiac sympathetic efferent nerve fibers (inhibition of activity) [9].

Autonomic regulation therapy by VNS in patients with heart failure has been shown to improve cardiac performance (left ventricular ejection fraction), to decrease left ventricular size (left ventricular systolic and diastolic diameter and volume), to reduce heart failure symptoms (NYHA class and quality of life), and favorably influence markers of risk for lethal arrhythmias [10].

The first-in-man single center study of VNS involved 8 patients and used the CardioFit 5000 device (BioControl Medical Ltd, Yehud, Israel), which is a closed loop system comprised of a proprietary bipolar electrode that is surgically implanted around the right vagal nerve, and a right ventricular sensing lead that allows for VNS to be synchronized so that the vagal nerve is stimulated after the QRS complex [11].

A different technical approach with an open loop VNS system that did not have a right ventricular sensing lead was used in the Autonomic Regulation Therapy via Left or Right Cervical Vague Nerve Stimulation in Patients with Chronic Heart Failure (ANTHEMHF) study [12]. Sixty patients with NYHA class II and III heart failure, left ventricle ejection fraction $<40 \%$, and $\mathrm{QRS}<150 \mathrm{~ms}$, who were randomized to either left or right cervical VNS were enrolled. The stimulation intensity of VNS was up-titrated during a 10-week period to reach $2.0 \pm 0.6 \mathrm{~mA}$. There was a not statistically significant trend toward greater improvement with right VNS. Overall, $77 \%$ of patients improved by at least one NYHA class at 6 months, with a significant improvement of quality of life [12].

According with NECTAR-HF (Neural Cardiac Therapy for Heart Failure) study VNS for the treatment of heart failure patients is safe and feasible [13]. This study enrolled patients with NYHA class II and III heart failure, a left ventricle ejection fraction $<35 \%$, a $\mathrm{QRS}<130 \mathrm{~ms}$, and left ventricle end-diastolic diameter $>55 \mathrm{~mm}$. All 96 enrolled patients received a device implant and were randomized 2:1 to active treatment or sham treatment (with activation of the device only during the titration visits) for the first 6 months; thereafter, all patients received active treatment. The investigators were used a helical bipolar electrode (with stimulation intensity of $1.4 \pm 0.8 \mathrm{~mA}$ ), without an intracardiac right ventricular sensing lead. Therefore was not possible any regulation of stimulation on the basis of heart rate. All patients improved significantly quality of life and the NYHA class.

Another small multicenter study with 32 patients with left ventricular ejection fraction $<35 \%$ and a history of chronic heart failure in symptomatic NYHA class II-III showed that vagal stimulation in heart failure patients is feasible and safe [14]. Chronic vagal stimulation was associated with improved quality of life and 6-min walk test, reduced left ventricular systolic volumes and improved left ventricular ejection fraction.

It is difficult to compare the results of these small studies because each study used a different device and stimulation protocol and obviously a different stimulation intensity and frequency. The stimulation intensity varied in the 3 studies being $1.3 \pm 08 \mathrm{~mA}$ in NECTAR-HF (range, 0.3-3.5), 2.0 $\pm 0.6 \mathrm{~mA}$ in ANTHEM-HF (maximum intensity, $3 \mathrm{~mA}$ ), and $4.2 \pm 1.2 \mathrm{~mA}$ in the CardioFit pilot trial (range, 1.1-5.5). However lower frequencies allow greater amplitudes to be reached with tolerable side effects. With increases in stimulation intensity, the number of recruited vague nerve fibers is progressively higher. It seems that intensities such as those used in the NECTAR-HF study, albeit provide an improvement of left ventricular systolic function, recruit only a minority of the fibers in the cervical vague trunk [15]. Probably the lower intensity of VNS in NECTAR-HF and ANTHEM-HF was not sufficient to adequately activate vagal fibers.

Increase of Vagal Tone in Heart Failure (INOVATE-HF) study, an international, multicenter, randomized clinical trial, assessed safety and efficacy of VNS using the CardioFit system in patients with symptomatic heart failure who are on guideline-directed medical therapy [16]. INOVATE-HF trial randomized 707 patients with NYHA class III symptoms, an left ventricular ejection fraction $\leq 40 \%$ and left ventricular end-diastolic dimensions 50 to 80 
$\mathrm{mm}$ in a 3:2 ratio to either active treatment (implanted) or continuation of medical therapy (not implanted). The mean follow-up was 16 months. Quality of life, NYHA functional class, and 6-min walking distance were favorably affected by VNS, but left ventricular endsystolic volume index was not different [17]. The authors concluded that VNS does not reduce the rate of death or heart failure events in chronic heart failure patients [17].

New techniques (e.q. quantitative Poincaré analysis) were developed in patients with heart failure who received chronic autonomic regulation therapy with an implantable VNS system on either the right or left side [18]. Obtaining a tight coupling in beat-to-beat dynamics during VNS directly related to stimulation intensity could provide a useful measurement for confirming autonomic engagement. This new technique for detecting acute heart rate responses to VNS suggests that the recruitment of nerve fibers responsible for heart rate changes were substantially lower in NECTAR-HF than in pre-clinical models [18].

\section{The implantation procedure: technique and complications}

The implantable neuro-stimulator is introduced through a minim invasive surgical technique. An electrode cuff is placed around the right (or left cervical vague) aiming to stimulate both the vagal efferent and afferent fibers.

Following positioning under local anesthesia of an intracardiac sensing electrode, the right (or left) vague is exposed through a latero-cervical incision under general anesthesia, the appropriate size of the cuff electrode is determined and the cuff positioned [19]. The stimulation lead is then tunneled and connected with the intracardiac sensing electrode to a subcutaneous stimulator in the

\section{References}

1. 2013 ESC guidelines on cardiac pacing and cardiac resynchronization therapy: the task force on cardiac pacing and resynchronization therapy of the European Society of Cardiology (ESC). Developed in collaboration with the right (or left) subclavicular region. Prior to closure, a continuity test and heart rate reduction by stimulation test need to be performed to ensure proper device functioning.

Common device-related adverse events after VNS titration are transient mild dysphonia, cough, and oropharyngeal pain, which are similar for left- and right-side VNS [12]. The most serious implantation-associated adverse effect is infection [20]. Mortality events, as well as the episodes of acute cardiac decompensation are considered predictable by the investigators, considering the severity of heart failure. It seems that these are safety without major adverse events clearly related to the investigational device.

\section{Conclusion}

Autonomic nervous system imbalance in heart failure has received considerable attention during the last decades. Although a favorable long-term safety profile was found in human, improvements in the efficacy endpoints in patients with heart failure by VNS are controversially. However new randomized larger clinical studies are necessary to evaluate the clinical role of this new approach.

\section{Conflict of interest}

The authors declare that they have no competing interests.

\section{Funding}

This work was supported by the Project entitled: Expanding and upgrading an Atrial Fibrillation Treatment Research Center as a method of preventing heart failure by developing the research and development infrastructure.

European Heart Rhythm Association (EHRA). Europace 2013; 15(8):1070-1118.

2. De Ferrari GM, Stolen $C$, Tuinenburg $A E$, et al. Long-term vagal stimulation for heart failure: Eighteen month results from the NEural 
Cardiac TherApy foR Heart Failure (NECTARHF) trial. Int $J$ Cardiol 2017; pii:S01675273(17)31295-0.

3. Armour JA, Murphy DA, Yuan BX, Macdonald S, Hopkins DA. Gross and microscopic anatomy of the human intrinsic cardiac nervous system. Anat Rec 1997; 247:289-298.

4. Armour JA. Potential clinical relevance of the 'little brain' on the mammalian heart. Exp Physiol 2008; 93:165-176.

5. De Ferrari GM, Schwartz PJ. Vagus nerve stimulation: from pre-clinical to clinical application: challenges and future directions. Heart Fail Rev 2011; 16(2):195-203.

6. Li M, Zheng $\mathrm{C}$, Sato $\mathrm{T}$, Kawada $\mathrm{T}$, Sugimachi $M$, Sunagawa K. Vagal nerve stimulation markedly improves long-term survival after chronic heart failure in rats. Circulation 2004; 109(1):120-124.

7. Zhang Y, Popovic ZB, Bibevski S, et al. Chronic vagus nerve stimulation improves autonomic control and attenuates systemic inflammation and heart failure progression in a canine highrate pacing model. Circulation 2009; 2:692699.

8. Hamann JJ, Ruble SB, Stolen C, et al. Vagus nerve stimulation improves left ventricular function in a canine model of chronic heart failure. Eur J Heart Fail 2013; 15:1319-1326.

9. Schwartz PJ. Vagal stimulation for heart diseases: from animals to men. - An example of translational cardiology. Circ J 2011; 75(1):2027.

10. Schwartz PJ, La Rovere MT, De Ferrari GM, Mann DL. Autonomic modulation for the management of patients with chronic heart failure. Circ Heart Fail 2015; 8(3):619-628.

11. Schwartz PJ, De Ferrari GM, Sanzo A, et al. Long term vagal stimulation in patients with advanced heart failure: first experience in man. Eur J Heart Fail 2008; 10:884-891.
12. Premchand RK, Sharma K, Mittal $S$, et al. Autonomic regulation therapy via left or right cervical vagus nerve stimulation in patients with chronic heart failure: results of the ANTHEMHF trial. J Card Fail 2014; 20:808-816.

13. Zannad F, De Ferrari GM, Tuinenburg AE, et al. Chronic vagal stimulation for the treatment of low ejection fraction heart failure: results of the NEural Cardiac TherApy foR Heart Failure (NECTAR-HF) randomized controlled trial. Eur Heart J 2015; 36:425-433.

14. De Ferrari GM. Vagal stimulation in heart failure. J Cardiovasc Transl Res 2014; 7(3):310-320.

15. Castoro MA, Yoo PB, Hincapie JG, et al. Excitation properties of the right cervical vagus nerve in adult dogs. Exp Neurol 2011; 227:6268.

16. Hauptman PJ, Schwartz PJ, Gold MR, et al. Rationale and study design of the increase of vagal tone in heart failure study: INOVATE-HF. Am Heart J 2012; 163:954-962.e1.

17. Gold MR, Van Veldhuisen DJ, Hauptman PJ, et al. Vagus Nerve Stimulation for the Treatment of Heart Failure: The INOVATE-HF Trial. J Am Coll Cardiol 2016; 68(2):149-158.

18. Libbus I, Nearing BD, Amurthur B, Ken Knight $\mathrm{BH}$, Verrier RL. Quantitative evaluation of heartbeat interval time series using Poincaré analysis reveals distinct patterns of heart rate dynamics during cycles of vagus nerve stimulation in patients with heart failure. $J$ Electrocardiol 2017; pii: S0022-0736(17)3018130184.

19. De Ferrari GM, Crijns HJ, Borggrefe $M$, et al. Chronic vagus nerve stimulation: a new and promising therapeutic approach for chronic heart failure. Eur Heart J 2011; 32(7):847-855.

20. Akdemir B, Benditt DG. Vagus nerve stimulation: An evolving adjunctive treatment for cardiac disease. Anatol J Cardiol 2016; 16(10):804-810. 